\title{
Nonlocal potentials for short-range electronic correlation in atoms, molecules, and solids
}

\author{
R. K. Nesbet \\ Physics Department, University of Connecticut, Storrs, CT 06269-3046, USA \\ Permanent address: IBM Almaden Research Center, 650 Harry Road, San Jose, CA 95120-6099, USA
}

(December 12, 2018)

For Int.J.Quantum Chem.

Extending density functional theory (DFT) to an ab initio orbital functional theory (OFT) requires new methodology for nonlocal exchange and correlation potentials. This paper describes such modifications to a standard Dirac-Slater atomic program. Unrestricted Hartree-Fock (UHF) theory is extended by a modified Colle-Salvetti Ansatz for short-range electronic correlation. Results are reported for atoms HeNe. Values of parameters needed for similar calculations on molecules and solids are reported. Implementation of nonlocal exchange and correlation for such extended systems, using multiple scattering theory to connect independent calculations in space-filling atomic cells, is discussed.

\section{INTRODUCTION}

Without invoking the electronic density as an intermediate, variational equations for an independent-electron model can be derived as an orbital functional theory (OFT) [1], in agreement with Kohn-Sham equations in the local density approximation (LDA). In contrast to density functional theory (DFT) $[2,3]$, an exact energy functional for OFT can be derived from many-body theory $[4,5]$. The orbital Euler-Lagrange equations (OEL) of OFT are determined by orbital functional derivatives, which take the general form of linear operators acting on occupied orbital functions of a model state. Because the exclusion principle requires independent normalization of the orbital partial densities, derivation of the OEL equations requires orbital wave functions or densities to be varied independently [6]. For $\mathrm{N}$ electrons, there are $\mathrm{N}$ normalization constraints. Independent Lagrange multipliers (the orbital energy eigenvalues) are determined by these normalization constraints, as they are for HartreeFock and Kohn-Sham equations.

In a density-based theory that incorporates the exclusion principle [7], Euler-Lagrange equations for the orbital densities are determined by partial (Gâteaux [8]) density functional derivatives, indexed by the orbital densities. Unless they are independent of the orbital index, these functional derivatives do not determine a unique total (Fréchet [8]) density functional derivative, equivalent to a multiplicative local potential function $[6,9]$. The required Gâteaux functional derivatives can be constructed from the corresponding orbital functional derivatives of OFT. The implied generalized Thomas-Fermi equations for orbital partial densities are operationally equivalent to the OEL equations [7].

Unless all orbital energies are equal $[6,9]$, the OEL equations cannot be derived by a theory which considers only the total electronic density [10]. Equal orbital energies conflict with the exclusion principle for the lowest state of any compact system with more than one electron of each spin. This is easily verified in the example of the $1 s 2 s^{3} S$ state of an atom with two noninteracting electrons of the same spin. Independent variation of orbital densities, required by the exclusion principle, defines Gâteaux functional derivatives. It follows from this analysis that extension of Kohn-Sham theory beyond the LDA cannot be done in general without introducing nonlocal potentials [10]. The required nonlocal potentials are well-defined in OFT, formulated as an application of the many-body theory of electrons.

The Schrödinger kinetic energy operator $\hat{t}=-\frac{1}{2} \nabla^{2}$ and the Fock exchange operator are well-known nonlocal "potentials" in standard theory. The present work includes nonlocal potentials for short-range correlation based on the correlation energy Ansatz of Colle and Salvetti [11]. The plan being followed is to implement these nonlocal potentials for calculations within atomic cells, then to use energy-linearized variational multiple scattering theory to match such local calculations together in large molecules and solids [12]. This methodology is outlined here. Unrestricted Hartree-Fock (UHF) theory can be implemented for atoms or atomic cells by modifying any standard local-potential atomic Slater or Kohn-Sham program, to replace local exchange by the nonlocal Fock operator. A similar modification allows inclusion of the correlation energy functional considered here. Total and orbital energies computed with this nonlocal exchangecorrelation model are reported for ground states of atoms He through Ne.

\section{NONLOCAL POTENTIALS IN ORBITAL FUNCTIONAL THEORY}

The simplest example of a nonlocal functional derivative is provided by the Schrödinger kinetic energy orbital functional

$$
T=\sum_{i} n_{i}(i|\hat{t}| i) .
$$

The notation here sums over occupied orbitals $\phi_{i}$ of a Slater-determinant reference state $\Phi$, with occupation 
numbers $n_{i}=1$ for $i \leq N$. Spin indices and sums are assumed, but suppressed in the notation. The total electronic density of the model state $\rho(\mathbf{r})=\sum_{i} n_{i} \rho_{i}(\mathbf{r})$ is a sum of orbital densities $\rho_{i}(\mathbf{r})=\phi_{i}^{*}(\mathbf{r}) \phi_{i}(\mathbf{r})$. The partial (Gâteaux) density functional derivative

$$
\frac{\delta T}{n_{i} \delta \rho_{i}}=v_{t i}(\mathbf{r})=\frac{\phi_{i}^{*} \hat{t} \phi_{i}}{\phi_{i}^{*} \phi_{i}}
$$

is determined as an orbital-indexed local potential function $[7,10]$ by the orbital functional derivative

$$
\frac{\delta T}{n_{i} \delta \phi_{i}^{*}}=\hat{t} \phi_{i}
$$

Applied to the functional $V=\sum_{i} n_{i}(i|v| i)$, for an external local potential $v(\mathbf{r})$, this analysis determines the Gâteaux derivative $v_{v i}(\mathbf{r})=v(\mathbf{r})$. Since this is independent of the orbital index, it reduces to a Fréchet derivative, a multiplicative local potential. The implied generalized Thomas-Fermi equations for orbital densities $i \leq N$ of noninteracting electrons are [7]

$$
v_{t i}(\mathbf{r})=\epsilon_{i}-v(\mathbf{r})
$$

The Lagrange multipliers $\epsilon_{i}$ are to be determined such that $\int d^{3} \mathbf{r} \rho_{i}=1$ for each orbital density. These equations are operationally equivalent to the noninteracting KohnSham or OEL equations [7]

$$
\hat{t} \phi_{i}=\left\{\epsilon_{i}-v(\mathbf{r})\right\} \phi_{i}
$$

Two-electron functionals are defined by $U=E_{h}+E_{x}$, where

$E_{h}=\frac{1}{2} \sum_{i, j} n_{i} n_{j}(i j|u| i j) ; E_{x}=-\frac{1}{2} \sum_{i, j} n_{i} n_{j}(i j|u| j i)$

and $u=1 / r_{12}$. The Hartree functional $E_{h}$ is an integral of an explicit function of total density, which determines the Coulomb potential $v_{h}(\mathbf{r})$ as a Fréchet derivative. Similarly, if $E_{x c}$ is defined as in the LDA, the present analysis determines a Fréchet functional derivative, and verifies the LDA Kohn-Sham equations. The orbital functional derivative of the exchange functional $E_{x}$ defines the Fock exchange operator $\hat{v}_{x}$ such that $\hat{v}_{x} \phi_{i}=-\sum_{j} n_{j}(j|u| i) \phi_{j}$. The functional derivative of $U=E_{h}+E_{x}$ defines $\hat{u}=v_{h}(\mathbf{r})+\hat{v}_{x}$. Explicitly,

$$
\frac{\delta U}{n_{i} \delta \phi_{i}^{*}}=\hat{u} \phi_{i}=\sum_{j} n_{j}(j|\bar{u}| j) \phi_{i}
$$

where $\bar{u}=u\left(1-\mathcal{P}_{12}\right)$, and $\mathcal{P}$ is the exchange operator for equal spin indices.

Given $(H-E) \Psi=0$ for an N-electron eigenstate and any rule $\Psi \rightarrow \Phi$ that determines a model state $\Phi$, unsymmetric normalization $(\Phi \mid \Psi)=(\Phi \mid \Phi)=1$ implies $E=$ $(\Phi|H| \Psi)=E_{0}+E_{c}$. Here $E_{0}=(\Phi|H| \Phi)=T+U+V$ is an explicit orbital functional, and $E_{c}=(\Phi|H| \Psi-\Phi)$ defines the correlation energy. If $\mathcal{Q}=I-\Phi \Phi^{\dagger}, E_{c}=$ $(\Phi|H| \Psi-\Phi)=(\Phi|H| \mathcal{Q} \Psi)$. This implies an exact but implicit orbital functional [5]

$$
E_{c}=-\left(\Phi\left|H\left[\mathcal{Q}\left(H-E_{0}-E_{c}-i \eta\right) \mathcal{Q}\right]^{-1} H\right| \Phi\right),
$$

for $\eta \rightarrow 0+$. In practice, some parametrized approximate $E_{c}$ must be used, defining a correlation potential operator such that

$$
\frac{\delta E_{c}}{n_{i} \delta \phi_{i}^{*}}=\hat{v}_{c} \phi_{i}
$$

This defines an indexed local potential, the Gâteaux derivative

$$
\frac{\delta E_{c}}{n_{i} \delta \rho_{i}}=v_{c i}(\mathbf{r})=\frac{\phi_{i}^{*} \hat{v}_{c} \phi_{i}}{\phi_{i}^{*} \phi_{i}} .
$$

Defining universal functional $F=E-V=T+U+E_{c}$, and an operator $\mathcal{F}=\hat{t}+\hat{u}+\hat{v}_{c}$, the general OEL equations are

$$
\frac{\delta F}{n_{i} \delta \phi_{i}^{*}}=\mathcal{F} \phi_{i}=\left\{\epsilon_{i}-v(\mathbf{r})\right\} \phi_{i}
$$

reducing in the LDA to Kohn-Sham equations. The OEL equations imply generalized Thomas-Fermi equations [7],

$$
\frac{\phi_{i}^{*} \mathcal{F} \phi_{i}}{\phi_{i}^{*} \phi_{i}}=v_{f i}(\mathbf{r})=\epsilon_{i}-v(\mathbf{r}) .
$$

If $\mathcal{F}$ is hermitian, the indexed potential $v_{f i}$ is the Gâteaux derivative $\delta F / n_{i} \delta \rho_{i}$. There is no implication in general that $E_{x}+E_{c}$ defines a Fréchet derivative.

\section{PLAN FOR LARGE MOLECULES AND SOLIDS}

The general case of nonlocal potentials determined explicitly by the idempotent Dirac density matrix

$$
\hat{\rho}(1,2)=\sum_{i} \phi_{i}\left(\mathbf{r}_{1}\right) n_{i} \phi_{i}^{*}\left(\mathbf{r}_{2}\right)
$$

is considered. In full-potential multiple scattering theory (MST) [13], local basis functions are constructed by integrating the Schrödinger or semirelativistic equation for specified orbital angular momentum $\ell$ and energy $\epsilon$ within the enclosing sphere $\left(r=r_{1}\right)$ of each atomic cell. Following ideas of canonical energy-band theory $[14,15]$, reviewed by Skriver [16], the energy-dependent radial wave function $u_{\ell}(\epsilon ; r)$ is characterized by its logarithmic derivative $D_{\ell}(\epsilon)=r_{S} u_{\ell}^{\prime}\left(r_{S}\right) / u_{\ell}\left(r_{S}\right)$, evaluated at the radius of a sphere whose volume equals that of a polyhedral atomic cell. As discussed originally by Wigner and Seitz, this cellular wave function can continue smoothly across a cell interface if $D_{\ell}(\epsilon)$ is negative, implying that $D_{\ell}\left(\epsilon_{B}\right)=0$ and $D_{\ell}\left(\epsilon_{A}\right)=-\infty$ define the lower and upper energy limits of an energy band. The band center is 
estimated to occur at $D_{\ell}\left(\epsilon_{C}\right)=-\ell-1$. An equivalent parameter is $p_{\ell}(\epsilon)=\frac{D_{\ell}(\epsilon)+\ell+1}{D_{\ell}(\epsilon)-\ell}$, which varies nearly linearly over the width of a band. It is found that orbital wave functions are well-approximated by linear interpolation over energies $\epsilon_{B} \leq \epsilon \leq \epsilon_{A}$.

This behavior indicates that an initial self-consistent calculation should be carried out for spherically averaged potentials, subject to the orbital boundary condition $D_{\ell}(\epsilon)=-\ell-1$ at $r_{S}$, in each inequivalent atomic cell. Because they satisfy a fixed boundary condition, the set of eigenfunctions $u_{\ell}\left(\epsilon_{C} ; r\right)$ can be extended to completeness within $r_{S}$, defining a local basis set for the linearized variational cellular method (LVCM) [12]. The computed nonlocal potential must be extended out to the enclosing sphere $r=r_{1}$, using the density matrix $\hat{\rho}$ constructed from the self-consistent cell orbitals, normalized to unity within $r_{S}$. Orbital functions should be computed, using this fixed potential, for energies $\epsilon_{C}$. Energy-derivative functions $\dot{u}_{\ell}\left(\epsilon_{C} ; r\right)$ must be computed as a basis for expanding the LVCM global matching function $[12,16]$.

At the UHF level of calculation, in each self-consistent iteration an average local exchange potential (Slater exchange) is defined by

$$
v_{x}(\mathbf{r})=\sum_{i} n_{i} v_{x i}(\mathbf{r}) \rho_{i}(\mathbf{r}) / \sum_{i} n_{i} \rho_{i}(\mathbf{r}),
$$

an exact formula if $v_{x i}$ reduces to a Fréchet derivative. This approximation is corrected by incremental inhomogeneous terms

$$
\left\{\hat{v}_{x}-v_{x}\right\} \phi_{i}
$$

evaluated from the previous iteration. This procedure converts a local-potential algorithm into UHF, and is valid for the indexed correlation potential considered below.

\section{SHORT-RANGE CORRELATION ENERGY}

Electronic correlation energy arises from two quite different sources. At distances larger than an atomic radius, multipolar response produces correlation effects evident in polarization potentials and dispersion forces. Longrange correlation, not considered here, requires computation of first-order multipole response pseudostates for each basis function $u_{\ell}\left(\epsilon_{C} ; r\right)$ [4]. A different approach is required for the short-range correlation due to the singularity of the interelectronic Coulomb potential $u=1 / r_{12}$. Expansion in $r_{12}$ about such a singularity shows that an N-electron wave function must have specific cusp behavior $[17,18]$ in order to cancel the singularity. The wave function must vary as $1+\frac{1}{2} r_{12}+\cdots$. Colle and Salvetti (CS) [11] impose this cusp condition through a symmetrical factor

$$
\Pi_{i<j}\left[1-\xi\left(\mathbf{r}_{i}, \mathbf{r}_{j}\right)\right]
$$

multiplying an antisymmetric model wave function. Using coordinates $\mathbf{q}=\mathbf{r}_{i}-\mathbf{r}_{j}$ and $\mathbf{r}=\frac{1}{2}\left(\mathbf{r}_{i}+\mathbf{r}_{j}\right)$ the CS Ansatz is

$$
\xi(\mathbf{r}, \mathbf{q})=\exp \left(-\beta^{2} q^{2}\right)\left[1-\Gamma(\mathbf{r})\left(1+\frac{1}{2} q\right)\right]
$$

This ensures the limiting forms

$$
\begin{aligned}
\left.(1-\xi)\right|_{q \rightarrow 0} & =\Gamma(\mathbf{r})\left(1+\frac{1}{2} q+\cdots\right) \\
\left.\xi\right|_{q \rightarrow \infty} & =0 .
\end{aligned}
$$

In adapting this Ansatz to orbital functional theory (OFT) $[1,5]$, it is desirable to retain the unsymmetric normalization condition $(\Phi \mid \Psi)=(\Phi \mid \Phi)=1$, where $\Psi$ is the correlated state, and $\Phi$ is a reference state Slater determinant. Then the correlation energy is $E_{c}=(\Phi|H| \Psi-\Phi)=$ $\sum_{i<j} n_{i} n_{j} E_{i j}$, a sum of electron-pair correlation energies. This suggests parametrization for each pair of occupied orbital functions $\phi_{i}, \phi_{j}$, such that

$$
E_{i j}=-\left(i j\left|\bar{u} \xi_{i j}(q)\right| i j\right)
$$

A parametrized form similar to $\mathrm{CS}$ is

$$
\xi_{i j}(q)=\exp \left(-\beta_{i j}^{2} q^{2}\right)\left[1-\gamma_{i j}\left(1+\frac{1}{2} q\right)\right]
$$

Parameter $\gamma_{i j}$ is determined by the normalization condition $(\Phi \mid \Psi-\Phi)=0$, or $\left(i j\left|\xi_{i j}\right| i j\right)=0$ for each pair:

$$
\gamma_{i j}=\frac{\left(i j\left|\exp \left(-\beta_{i j}^{2} q^{2}\right)\right| i j\right)}{\left(i j\left|\exp \left(-\beta_{i j}^{2} q^{2}\right)\left(1+\frac{1}{2} q\right)\right| i j\right)} .
$$

The free parameter $\beta_{i j}$ can be chosen to minimize BetheGoldstone (BG or IEPA) energy [19] for specified $i, j$, or can be treated as a semiempirical parameter and fitted to known correlation energies of atoms.

This Ansatz can be incorporated into OFT by using Eq.(19) to define the model correlation energy. At any stage of the self-consistency iteration, parameter $\gamma_{i j}$ is determined by the consistency condition given above, and $\beta_{i j}$ is either a fixed empirical parameter, or is to be updated by solving a 2-electron BG equation indexed by orthogonal occupied orbitals $i j$. The indexed local correlation potential (Gâteaux functional derivative) is [10]

$$
v_{c i}(\mathbf{r})=\frac{\phi_{i}^{*}(\mathbf{r}) \hat{v}_{c} \phi_{i}(\mathbf{r})}{\phi_{i}^{*}(\mathbf{r}) \phi_{i}(\mathbf{r})}
$$

where

$$
\hat{v}_{c} \phi_{i}=\frac{\delta E_{c}}{n_{i} \delta \phi_{i}^{*}}=-\sum_{j} n_{j}\left(j\left|\bar{u} \xi_{i j}\right| j\right) \phi_{i},
$$

a direct generalization of the operator $\hat{u}$. Both $v_{h}$ and $\hat{v}_{x}$ are modified by short-range correlation, and antisymmetry is built in. Because terms $j=i$ vanish, this Ansatz for Coulomb-cusp correlation does not produce 
self-interaction. The indexed local potential $v_{c i}(\mathbf{r})$ is singular at nodes of $\phi_{i}(\mathbf{r})$. As in Hartree-Fock methodology, $\hat{v}_{c} \phi_{i}$ should be treated as an inhomogeneous term in numerical solution of the orbital Euler-Lagrange (OEL) equations.

Coupling of electron pairs is significant in standard CI methods for electronic correlation [19]. Although such coupling is inherent in the self-consistency of the proposed mean-field model, a more accurate extension of the method may be needed. A possible procedure is to implement the coupled electron-pair approximation (CEPA) [20] within each atomic cell, using the modified CS Ansatz as a closure formula for the CI expansion.

\section{A. Notes on integrals}

The normalized orbital basis functions are of the form

$$
\phi_{a}(\mathbf{r})=N_{a} \chi_{a}(r) Y_{\ell_{a} m_{a}}(\theta, \phi),
$$

where $r \chi_{a}(r)$ is a numerical solution of the radial OEL equation. The normalization constants are $N_{a}=$ $\left[\int r^{2} d r \chi_{a}^{2}(r)\right]^{-\frac{1}{2}}$. Definite and indefinite integrals are required for two-electron generalized potential functions $F(q)$, where $q^{2}=r_{1}^{2}+r_{2}^{2}-2 r_{1} r_{2} \cos \theta$. Integration over angles follows the standard derivation [21] of Condon and Shortley for $F(q)=1 / q$. For normalized radial functions,

$$
(a b|F| c d)=\sum_{k} c^{k}(a c) c^{k}(d b) F^{k}(a c ; d b),
$$

where $c^{k}$ denotes a Gaunt coefficient [21] and

$$
\begin{gathered}
F^{k}(a c ; d b)=\int_{0}^{\infty} r_{1}^{2} d r_{1} \int_{0}^{\infty} r_{2}^{2} d r_{2} \\
f^{k}\left(r_{1}, r_{2}\right) \chi_{a}^{*}\left(r_{1}\right) \chi_{c}\left(r_{1}\right) \chi_{d}\left(r_{2}\right) \chi_{b}^{*}\left(r_{2}\right) .
\end{gathered}
$$

The factor $f^{k}$ is

$$
f^{k}\left(r_{1}, r_{2}\right)=\frac{2 k+1}{2} \int_{0}^{\pi} \sin \theta d \theta P_{k}(\cos \theta) F(q) .
$$

Given $r_{1}$ and $r_{2}, \cos \theta=\frac{r_{1}^{2}+r_{2}^{2}-q^{2}}{2 r_{1} r_{2}}$ and $\sin \theta d \theta=\frac{q d q}{r_{1} r_{2}}$. In internal coordinates, $f^{k}\left(r_{1}, r_{2}\right)$ is an integral of the form

$$
\frac{2 k+1}{2 r_{1} r_{2}} \int_{\left|r_{1}-r_{2}\right|}^{r_{1}+r_{2}} P_{k}\left(\frac{r_{1}^{2}+r_{2}^{2}-q^{2}}{2 r_{1} r_{2}}\right) q F(q) d q .
$$

These integrals are needed for $q F(q)$ equal to $1, q, q^{2}$ times the factor $\exp \left(-\beta^{2} q^{2}\right)$, so that the integrand of $f^{k}$ is this Gaussian factor times a polynomial in $q$. It can easily be verified that $f^{k}$ reduces to $r_{<}^{k} / r_{>}^{k+1}$ for $\beta \rightarrow 0$ if $q F(q)=1$. The Legendre polynomial factors of the integrand are determined by the recurrence formula

$$
P_{k+1}(x)=\frac{2 k+1}{k+1} x P_{k}(x)-\frac{k}{k+1} P_{k-1}(x),
$$

with $P_{-1}(x)=0, P_{0}(x)=1$. The elementary integrals required are of the form $I_{n}(\beta ; \kappa)=\int_{0}^{\kappa} e^{-\beta^{2} q^{2}} q^{n} d q$. By a change of variables such that $t=\beta^{2} q^{2}$, this reduces to

$$
\begin{aligned}
I_{n}(\beta ; \kappa) & =\frac{1}{2 \beta^{n+1}} \int_{0}^{\beta^{2} \kappa^{2}} e^{-t} t^{\frac{1}{2}(n-1)} d t \\
& =\frac{\Gamma\left(\frac{1}{2}(n+1)\right)}{2 \beta^{n+1}} p\left(\frac{1}{2}(n+1), \beta^{2} \kappa^{2}\right),
\end{aligned}
$$

in terms of the incomplete gamma function $p(a, x)=$ $1-\Gamma(a, x) / \Gamma(a)[22]$, Sect.6.2. $p(a, x)$ can be computed effficiently using a power series for small $x$ and a continued fraction for large $x$. The continued fraction terminates if $n$ is odd.

\section{CALCULATIONS ON LIGHT ATOMS}

For applications to molecules and solids, using multiple scattering theory and an atomic cell model, values of the parameters $\beta_{i j}$ can be obtained by calculations on atoms. Results of such calculations, for light atoms $\mathrm{He}$ through Ne, are reported here. A numerical Dirac-Slater program [23] was modified as described above for UHF (exchange-only) and OFT calculations, the latter incorporating the modified CS correlation energy functional described above. The program was used in its nonrelativistic mode. Angular coefficients in the total energy functional were computed such that effective potentials are spherically averaged, but retain a spin index. This equivalence restriction implies that radial orbital functions with indices $\ell, m_{s}$ are well-defined.

For each atom considered, three sets of self-consistent calculations were carried out: for the He-like ion, for the Be-like ion (for $N \geq 4$ ), and for the neutral atom. To verify the computational method, computed UHF energies are compared in Table(I) with established RHF energies [24] and with total energies including correlation [25]. The OFT calculations were used to determine $\beta_{i j}$ parameters such that the computed total energies agreed with the "experimental" values shown in Table(I). Pairindexed parameters $\beta_{1 s 1 s}$ were determined for He-like ions, parameters $\beta_{2 s 2 s}$ for Be-like ions, and parameters $\beta_{2 p 2 p}$ for the neutral atoms. In each case, inner parameters were frozen and intershell parameters such as $\beta_{1 s 2 s}$ were scaled to the geometric mean of the corresponding intrashell parameters. Values of the latter that fit total energies of the ions and atoms considered are listed in Table(II). These parameters are the principal result of the present calculations, intended to define parametrized correlation functionals for extended systems.

Self-consistent UHF orbital energies are tabulated in Table(III) and OFT orbital energies in Table(IV). Because Janak's theorem [26] is valid in OFT, these energies have a physical meaning in the context of a theory in which orbital occupation numbers are allowed to change continuously and to have fractional values. They are 
derivatives of the total energy with respect to infinitesimal changes of the occupation numbers. Physical energy differences, by implication, correspond to integrals of these derivatives over finite increments of occupation numbers.

\section{CONCLUSIONS}

This paper considers an independent-electron model that incorporates a theoretically motivated Ansatz for correlation energy, expressed as a parametrized orbital functional. Computed results extend exchange-only theory (UHF) to a formalism parametrized by exact atomic ground-state energies. Parameters are obtained that make it possible to apply this formalism to calculations of the electronic structure of molecules and solids.

\section{ACKNOWLEDGMENTS}

This work was initiated at the University of Connecticut, supported by a grant from the University of Connecticut Research Foundation. The author is grateful to H. H. Michels for discussions of the new methodology proposed here, and to Prof. Michels and Prof. Wm. C. Stwalley for encouraging and implementing a visiting appointment at Storrs.

[1] Nesbet, R.K., Int.J.Quantum Chem. 2001, 81, 384.

[2] Hohenberg, P. and Kohn, W. Phys.Rev. 1964, 136, B864.

[3] Kohn, W. and Sham, L.J., Phys.Rev. 1965, 140, A1133.

[4] Nesbet, R.K., Phys.Rev.A 2000, 62, 040701(R).

[5] Nesbet, R.K., Int.J.Quantum Chem. 2001, 85, 405.

[6] Nesbet, R.K., Phys.Rev.A 2002, 65, 010502(R).

[7] Nesbet, R.K.. Int.J.Quantum Chem. 2002 90, 262.

[8] Blanchard, P. and Brüning, E., Variational Methods in Mathematical Physics: A Unified Approach; SpringerVerlag: Berlin, 1992 .

[9] Nesbet R.K., Phys.Rev.A 1998, 58, R12.

[10] Nesbet, R.K., Int.J.Quantum Chem. 2003 95, xxx. Published online 12 June 2003.

[11] Colle, R. and Salvetti, O., Theoret.Chim.Acta 1975 37, 329.

[12] Nesbet, R.K., Int.J.Quantum Chem. 2003 91, 46.

[13] Gonis, A. and Butler, W.H., Multiple Scattering in Solids, (Springer, New Yorki, 2000).

[14] Andersen, O.K., Phys.Rev.B 1975 12, 3060.

[15] Andersen, O.K. and Jepsen, O., Physica B 1977 91, 317.

[16] Skriver, H.L., The LMTO Method, (Springer-Verlag, Berlin, 1984).

[17] Kato, T., Commun.Pure Appl.Math. 1957 10, 151.

[18] Bingel, W.A., Theoret.Chim.Acta 1967 8, 54.
[19] Szabo. A. and Ostlund, N. S., Modern Quantum Chemistry; Introduction to Advanced Electronic Structure Theory, (McGraw-Hill, New York, 1989), revised first edition.

[20] Meyer, W., J.Chem.Phys. 1973 58, 1017.

[21] Condon, E.U. and Shortley, G.H., The Theory of Atomic Spectra, (Cambridge Univ. Press, New York, 1935).

[22] Press, W. H., Teukolsky, S. A., Vetterling, W. T., and Flannery, B. P., Numerical Recipes. (Cambridge Univ. Press, New York, 1992).

[23] Desclaux, J. P., Dirac-Slater program package incl. RESLD, (CEA, Paris, 1969, unpublished).

[24] Clementi, E. and Roetti, C., At.Data Nucl.Data Tables $197414,177$.

[25] Clementi, E., J.Chem.Phys 1963 38, 2248.

[26] Janak, J.F., Phys.Rev.B 1978 18, 7165. 
TABLE I. Total energies in Hartree units

\begin{tabular}{lrrr}
\hline \hline Atom & RHF & UHF & exp \\
\hline $\mathrm{He}$ & -2.8617 & -2.8617 & -2.9038 \\
$\mathrm{Li}$ & -7.4327 & -7.4328 & -7.4780 \\
$\mathrm{Be}$ & -14.5730 & -14.5730 & -14.6674 \\
$\mathrm{~B}$ & -24.5291 & -24.5293 & -24.6541 \\
$\mathrm{C}$ & -37.6886 & -37.6900 & -37.8466 \\
$\mathrm{~N}$ & -54.4009 & -54.4045 & -54.5890 \\
$\mathrm{O}$ & -74.8094 & -74.8136 & -75.0674 \\
$\mathrm{~F}$ & -99.4093 & -99.4108 & -99.7333 \\
$\mathrm{Ne}$ & -128.5470 & -128.5470 & -128.9400 \\
\hline \hline
\end{tabular}

TABLE II. Orbital $\beta$ parameters

\begin{tabular}{lccc}
\hline \hline Atom & $1 \mathrm{~s}$ & $2 \mathrm{~s}$ & $2 \mathrm{p}$ \\
\hline $\mathrm{He}$ & 0.83455 & & \\
$\mathrm{Li}$ & 1.42494 & 1.16853 & \\
$\mathrm{Be}$ & 2.01812 & 0.52267 & \\
$\mathrm{~B}$ & 2.61268 & 0.71557 & 0.82167 \\
$\mathrm{C}$ & 3.20278 & 0.89701 & 1.08430 \\
$\mathrm{~N}$ & 3.79877 & 1.05841 & 1.39511 \\
$\mathrm{O}$ & 4.39600 & 1.20737 & 1.55724 \\
$\mathrm{~F}$ & 4.99306 & 1.34754 & 1.77434 \\
$\mathrm{Ne}$ & 5.57807 & 1.47974 & 2.00125 \\
\hline \hline
\end{tabular}

TABLE III. UHF orbital energies (Hartree units)

\begin{tabular}{lcccccc}
\hline \hline Atom & $1 s_{\alpha}$ & $1 s_{\beta}$ & $2 s_{\alpha}$ & $2 s_{\beta}$ & $2 p_{\alpha}$ & $2 p_{\beta}$ \\
\hline $\mathrm{He}$ & -0.91796 & -0.91796 & & & & \\
$\mathrm{Li}$ & -2.48668 & -2.46870 & -0.19637 & & & \\
$\mathrm{Be}$ & -4.73267 & -4.73267 & -0.30927 & -0.30927 & & \\
$\mathrm{~B}$ & -7.70036 & -7.68527 & -0.54022 & -0.44175 & -0.31671 & \\
$\mathrm{C}$ & -11.34480 & -11.29972 & -0.82450 & -0.57914 & -0.43818 & \\
$\mathrm{~N}$ & -15.67067 & -15.58098 & -1.16297 & -0.72580 & -0.57092 & \\
$\mathrm{O}$ & -20.70635 & -20.62807 & -1.41447 & -1.07069 & -0.67505 & -0.52144 \\
$\mathrm{~F}$ & -26.40567 & -26.35786 & -1.66855 & -1.47217 & -0.76666 & -0.67975 \\
$\mathrm{Ne}$ & -32.77237 & -32.77237 & -1.93040 & -1.93040 & -0.85041 & -0.85041 \\
\hline \hline
\end{tabular}

TABLE IV. OFT orbital energies (Hartree units)

\begin{tabular}{lcccccc}
\hline \hline Atom & $1 s_{\alpha}$ & $1 s_{\beta}$ & $2 s_{\alpha}$ & $2 s_{\beta}$ & $2 p_{\alpha}$ & $2 p_{\beta}$ \\
\hline $\mathrm{He}$ & -0.94667 & -0.94667 & & & & \\
$\mathrm{Li}$ & -2.52396 & -2.50794 & -0.19546 & & & \\
$\mathrm{Be}$ & -4.81661 & -4.81661 & -0.31196 & -0.31106 & & \\
$\mathrm{~B}$ & -7.80392 & -7.79277 & -0.53861 & -0.45076 & -0.32004 & \\
$\mathrm{C}$ & -11.46803 & -11.43171 & -0.81782 & -0.59356 & -0.44126 & \\
$\mathrm{~N}$ & -15.80892 & -15.73252 & -1.15024 & -0.74332 & -0.57450 & \\
$\mathrm{O}$ & -20.86288 & -20.79561 & -1.39967 & -1.08080 & -0.67795 & -0.54422 \\
$\mathrm{~F}$ & -26.58113 & -26.53950 & -1.65541 & -1.47252 & -0.77354 & -0.69690 \\
$\mathrm{Ne}$ & -32.96943 & -32.96943 & -1.92025 & -1.92025 & -0.86284 & -0.86284 \\
\hline \hline
\end{tabular}

